\title{
Feasibility of Asynchronous Learning in Collegiate EMS: Impact of a Novel Training Program on Self- Reported Measures of Confidence
}

\author{
Katherine R. Luchette, AEMT-Cardiac; James Dorroh, AAS, NRP; Gita Pensa, \\ MD; Megan L. Ranney, MD, MPH, FACEP; Thomas J. Martin, AEMT-Cardiac
}

\section{POSTER PRESENTATION ABSTRACT}

Introduction: Brown University EMS (BEMS) provides 24/7 emergency medical coverage using one non-transport SUV and one ambulance equipped at the BLS and ALS/BLS levels, respectively. Each vehicle must be attended by an ALS staff or BLS student supervisor per standard operating guidelines. While this dual-supervisor model allowed BEMS to capture $25 \%$ more calls in 2016, its maintenance requires a robust staff of qualified student supervisors. Though progression models for becoming a "supervisor" or "crew chief" vary by institution, the loss of experienced personnel following graduation is a ubiquitous challenge. Program Development \& Implementation: A novel BLS Supervisor Training Program (STP) was implemented during Fall 2017. Using asynchronous learning, we sought to optimize training time by promoting self-learning off-shift and outside of traditional didactic models. Concurrent with 48 hours/month of precepted field training, students participated in 16 weekly sessions, alternating between two hours of small-group, problem-based learning and online modules accessed through our internal website. Online content was developed internally using original exercises and augmented by Free Open Access Medical Education resources, including selected podcasts from EmCrit and Emergency Medicine Cases. Program Evaluation: As part of internal QI efforts, questionnaires assessing students' goals and progress were routinely included during STP. Students $(\mathrm{N}=7)$ rated their confidence at supervising BLS calls on a 0-10 scale ranging from "Not at all Confident" to "Absolutely Confident" with 100\% response rates. Mean [95\% CI] scores of 5.4 [3.9, 6.9] and 8.6 [8.0, 9.2] were collected pre- and post-STP, respectively, and there was significant improvement within individual scores (Paired t-test: $\mathrm{p}=0.004$, 95\% CI [1.4, 4.9]). Discussion/Conclusion: Student training and development within collegiate EMS may be limited by call volume, time-demands, and personnel turnover. We propose asynchronous learning as a feasible method to optimize training time in collegiate EMS through accommodating undergraduates' time constraints. Similar education models may increase operational efficiency, patient safety, and quality care.

Author Affiliations: From Emergency Medical Services, Brown University, Providence, RI, USA (K.R.L., J.D., T.J.M.); Program in Emergency Digital Health Innovation, Department of Emergency Medicine, The Warren Alpert Medical School of Brown University, Providence, RI, USA (G.P., M.L.R., T.J.M.).

Address for Correspondence: Thomas J. Martin, AEMT-Cardiac, The Warren Alpert Medical School of Brown University, 69 Brown St., Box 2784, Providence, RI, USA. E-mail: thomas_martin@brown.edu

Conflicts of Interest/Funding Sources: By the JCEMS Submission Declaration Form, all authors are required to disclose all potential conflicts of interest and funding sources. The authors declared that they have no conflicts of interest. The authors declared that they did not receive funding to conduct the program or research associated with this work.

Ethical Compliance: The authors attest that the research associated with this abstract was conducted in accordance with the JCEMS Ethics Guidelines.

Submission History: Received January 14, 2018; accepted for presentation and publication February 21, 2018.

Poster Presentation: This abstract was presented as a poster at the Academ- ic Poster Session of the $25^{\text {th }}$ Annual Conference of the National Collegiate Emergency Medical Services Foundation; February 24, 2018; Philadelphia, PA, USA. The authors received the Third Place Award for the Best Poster Presentation Competition. The poster is available for download on the JCEMS website.

Published Online: May 1, 2018

Published in Print: August 13, 2018 (Volume 1: Supplemental 1)

Reviewer Information: In accordance with JCEMS editorial policy, poster presentation abstracts undergo double-blind peer-review by at least two reviewers (JCEMS Editorial Board members and/or independent reviewers) prior to acceptance for presentation and publication. JCEMS thanks the anonymous reviewers who contributed to the review of this work.

Copyright: (c) 2018 Luchette, Dorroh, Pensa, Ranney, \& Martin. This is an open access abstract distributed under the terms of the Creative Commons Attribution 4.0 International (CC BY 4.0) License, which permits unrestricted use, distribution, and reproduction in any medium, provided the original author and source are credited. The full license is available at: https://creativecommons.org/licenses/by/4.0/

Electronic Link: https://doi.org/10.30542/JCEMS.2018.01.S1.03 